MATHEMATICS OF COMPUTATION

Volume 67, Number 221, January 1998, Pages 87-109

S $0025-5718(98) 00921-1$

\title{
EXISTENCE AND UNIQUENESS OF TRAVELING WAVES AND ERROR ESTIMATES FOR GODUNOV SCHEMES OF CONSERVATION LAWS
}

\author{
HAITAO FAN
}

\begin{abstract}
The existence and uniqueness of the Lipschitz continuous traveling wave of Godunov's scheme for scalar conservation laws are proved. The structure of the traveling waves is studied. The approximation error of Godunov's scheme on single shock solutions is shown to be $O(1) \Delta x$.
\end{abstract}

\section{INTRODUCTION}

The field equations expressing the balance laws for one-dimensional homogeneous continuous media typically have the form of systems of conservation laws

$$
u_{t}+f(u)_{x}=0, \quad x \in \mathbb{R}, \quad t>0, \quad u \in \mathbb{R}^{d} .
$$

In this paper, we shall investigate the existence of discrete traveling waves and error estimates in the presence of shocks for Godunov's scheme.

It is well known that in general the initial value problem of (1.1) develops discontinuities in a finite time. Numerical schemes for (1.1) typically have an error of $O(1)$ order near the shock which makes the error estimates difficult to obtain. For this reason, most theoretical results for numerical schemes are either estimates for smooth solutions or about the convergence of the schemes without stating the rate of convergence. To obtain error estimates for numerical schemes in the presence of shocks, we need to know the existence and stability of discrete traveling waves for numerical schemes, [Je], [LX1], [LX2]. Thus, a brief review of the known results on the existence and stability of discrete traveling waves of numerical schemes for conservation laws and error estimates of these schemes when shocks are present in the solutions is in order. Jennings [Je] proved the existence and stability of discrete traveling waves for strictly monotone schemes with differentiable fluxes for scalar conservation laws. The existence of discrete shock waves of first order accurate finite difference schemes for systems of conservation laws was established by Majda and Ralston [MR] by using the center manifold theorem, see also [Mi]. Engquist and Osher, [EO], proved the stability of their monotone scheme. Smyrlis [Sm] showed the stability of a stationary discrete shock for the Lax-Wendroff scheme. Szepessy [Sz] studied the $L^{2}$ stability of stationary discrete shock for a first order implicit streamline diffusion scheme. Liu and Xin proved $L^{1}$ and $L^{2}$ stability of discrete shocks, [LX1], and convergence to piecewise smooth solutions, [LX2], of the Lax-Friedrichs scheme for systems of conservation laws. $\mathrm{Yu}[\mathrm{Yu}]$ proved the

Received by the editor February 9, 1996 and, in revised form, August 19, 1996.

1991 Mathematics Subject Classification. Primary 65M10 35L65.

Research supported by NSF Fellowship under Grant DMS-9306064. 
existence of discrete shocks for a Lax-Wendroff scheme and studied the stability of discrete shocks for Lax-Wendroff schemes with small speed. The error estimates for numerical schemes for conservation laws were studied by a number of authors. Kuznetsov $[\mathrm{Ku}]$ showed that monotone schemes have an upper $L^{1}$ error bound of order $\sqrt{\Delta x}$. Tang and Teng [TT] later proved that the lower bound for the $L^{1}$ norm of the error of monotone schemes on linear conservation laws is also of order $\sqrt{\Delta x}$. For nonlinear scalar conservation laws, due to Jennings' results [Je] and that monotone schemes are $L^{1}$ contracting, the $L^{1}$ norm of the error of the scheme on a single shock solution can be shown to be of $\Delta x$ order. Later, Liu and Xin [LX1] showed that for single shock solutions of systems of conservation laws, the $L^{1}$ error of a Lax-Friedrichs scheme is of $\Delta x$ order except for an initial layer. Recently, Teng and Zhang [TZ] obtained the same $L^{1}$ error estimate for scalar conservation laws with piecewise constant initial data, allowing shock interactions. Engquist and $\mathrm{Yu}$ [EY] obtained local error estimates under some conditions which are satisfied by monotone schemes, the E-O scheme and numerically verified for the Lax-Wendroff scheme for scalar conservation laws with a Riemann like initial value. Their estimates show that the contribution of the shock to the error decays exponentially away from the shock and the error of the location of the computed shock is $O(1) \Delta x$.

Almost all of the above results are either for monotone schemes or for the LaxWendroff scheme. Monotone schemes are necessarily first order accurate which usually produce a smeared image of shocks while the Lax-Wendroff scheme is highly oscillatory and may violate entropy conditions. On the other hand, almost all useful high resolution schemes are adaptive and few analytical results involving shocks are available for these schemes even though some of them are widely used. This makes the study of issues such as the existence of discrete traveling waves and error estimates for these schemes and the development of tools that can handle these problems a necessity. Some of the major difficulties in analyzing these schemes are their adaptiveness and that their flux functions $\bar{f}_{j+1 / 2}$ are Lipschitz continuous rather than continuously differentiable. One starting point for attacking these issues is Godunov's scheme since it is the base for many higher resolution schemes. We note that Jennings' results in [Je] do not cover Godunov's scheme since his proof relies heavily on the strict monotonicity of the schemes and differentiability of the flux function.

In this paper, we consider the existence of discrete traveling waves and error estimates for Godunov's scheme

$$
u_{j}^{n+1}=u_{j}^{n}-\lambda\left(\bar{f}_{j+1 / 2}^{n}-\bar{f}_{j-1 / 2}^{n}\right),
$$

where $\lambda=\Delta t / \Delta x$ and

$$
\bar{f}_{j+1 / 2}^{n}=\left\{\begin{array}{l}
\min _{u_{j}^{n} \leq u \leq u_{j+1}^{n} f(u),} \text { if } u_{j}^{n} \leq u_{j+1}^{n}, \\
\max _{u_{j}^{n} \geq u \geq u_{J+1}^{n}} f(u), \quad \text { if } u_{j}^{n} \geq u_{j+1}^{n} .
\end{array}\right.
$$

Here, we assume the CFL stability condition $\lambda \max _{u \in\left[-2\left|u_{+}\right|, 2\left|u_{-}\right|\right]}\left|f^{\prime}(u)\right|<1$.

Let us denote the schemes by

$$
u_{j}^{n+1}=\left(G u^{n}\right)_{j} .
$$

We intend to establish the existence of the traveling wave of (1.3) that satisfies

$$
u_{j} \rightarrow u_{ \pm} \quad \text { as } j \rightarrow \pm \infty .
$$


The speed of the traveling wave is

$$
s=\frac{f\left(u_{+}\right)-f\left(u_{-}\right)}{u_{+}-u_{-}} .
$$

For convenience, we assume $f^{\prime \prime}>0$, and that $f^{\prime}(a)=0$ for some constant $a$. We assume that $u_{+}<a<u_{-}$because otherwise Godunov's scheme is reduced to the upwinding scheme for which G. Jennings [Je] already obtained results. A traveling wave solution of the numerical scheme (1.3) is a function, $u(x)$, defined on $\mathbb{R}^{1}$, satisfying

$$
\begin{aligned}
& u(x-\lambda s)=G u(x), \\
& \sum_{j=-\infty}^{\infty}\left[u(y+j)-\int_{j-1 / 2}^{j+1 / 2} u(y+x) d x\right]=0, \\
& u(x) \rightarrow u_{ \pm} \quad \text { as } x \rightarrow \pm \infty .
\end{aligned}
$$

For the case where $\lambda s=l / m$ where $l$ and $m$ are integers, the equation of the traveling wave is usually written as

$$
\begin{aligned}
& u_{j}^{k+1}=u_{j}^{k}-\lambda\left(\bar{f}_{j+1 / 2}\left(u^{k}\right)-\bar{f}_{j-1 / 2}\left(u^{k}\right)\right), \quad k=0,1,2, \ldots, m-1, \\
& u_{j}^{0}=u_{j}, \quad u_{j}^{m}=u_{j-l} \\
& u_{j} \rightarrow u_{ \pm} \quad \text { as } j \rightarrow \pm \infty .
\end{aligned}
$$

We define an operator $T$ by

$$
(T u)_{j-l}:=\left(G^{m} u\right)_{j} .
$$

Then the traveling wave equation (1.7) becomes

$$
\begin{aligned}
& u=T u, \\
& u_{j} \rightarrow u_{ \pm} \text {as } j \rightarrow \pm \infty .
\end{aligned}
$$

Our plan is to prove the existence of a one-parameter family of solutions of (1.6) for the case where $\lambda s$ is rational. Based on these solutions, we construct a Lipschitz continuous traveling wave solution, $u(x)$, of (1.5) for Godunov's scheme. We then prove the existence of traveling wave solutions, satisfying (1.5), for Godunov's scheme when $\lambda s$ is irrational.

We assume that $\lambda s \geq 0$. Then there is a point $b \in\left(a, u_{-}\right]$such that $f(b)=f\left(u_{+}\right)$. Our main results are as follows:

Theorem 1.1. If $f$ is convex and $u_{-} \geq u_{+}$, then there is a unique traveling wave solution $u(x)$, in the sense of (1.5), of Godunov's scheme which satisfies

$$
\begin{gathered}
\int_{\mathbb{R}}|u(x)-V(x)| \leq\left(3+\frac{\lambda s(1-\lambda s)}{2 \gamma}\right)\left|u_{+}-u_{-}\right|, \\
u(x) \geq b \quad \text { for } x \leq J(u), \\
u(x)=u_{+} \quad \text { for } x \geq J(u)+2
\end{gathered}
$$

for some $J(u) \in \mathbb{R}$ and where

$$
V(x)= \begin{cases}u_{-}, & \text {if } x \leq J(u)+1, \\ u_{+}, & \text {if } x>J(u)+1,\end{cases}
$$


and

$$
\left|u(x)-u_{-}\right| \leq \alpha \exp (-\beta|x-J(u)|) \quad \text { for } x<J(u)
$$

for some constant $\alpha, \beta>0$ depending on $\lambda s$ and $u_{ \pm}$. Moreover, the solution is Lipschitz continuous with Lipschitz constant $\left|u_{-}-u_{+}\right|$.

It is clear that for any fixed $x_{0}, u\left(x_{0}+j\right)$, where $u(x)$ is the traveling wave in the sense of (1.5) provided in Theorem 1.1, is a solution of (1.6) when $\lambda s$ is rational. The following theorem states that the form $u\left(x_{0}+j\right)$ includes all solutions of (1.6).

Theorem 1.2. Let $u(x)$ be the solution of (1.5) and $\lambda s$ be rational. Then any solution $v_{j}$ of (1.6) can be written as $u\left(x_{0}+j\right)$ for some $x_{0} \in \mathbb{R}$.

Remark 1. Statement (ii) of Theorem 1.1 indicates that the discrete shock profile remains constant, $u_{+}$, from a few grid points before the shock to infinity, while decay exponentially to $u_{-}$after the shock. From $j=J(u)+1$ to $J(u)$, the shock profile jumps from $u_{+}$to $b$. These phenomena do not occur for strictly monotone schemes. The resuts of Theorem 1.1 provide a key element for studying the error estimates near shocks for Godunov's scheme in the future.

Remark 2. We expect to prove, at least when $\lambda s$ is rational, that

$$
\sum_{j=-\infty}^{\infty}\left|\left(G^{n} v\right)_{j}-u\left(x_{0}+j-\lambda s n\right)\right| \rightarrow 0 \quad \text { as } n \rightarrow \infty
$$

by using Corollary 3.12 of this paper and Theorem A of [OR]. This is left to further investigations.

Remark 3. Without restriction $(1.5)_{2}$, solutions $u(x)$ to (1.5) are not unique, up to shifts, when $\lambda s$ is rational.

We consider the initial value problem (1.1) with

$$
u(x, 0)= \begin{cases}u_{-}, & \text {if } x<x_{0} \\ u_{+}, & \text {if } x>x_{0}\end{cases}
$$

where $u_{-}>u_{+}$. The solution of (1.14) is the shock

$$
w(x, t)= \begin{cases}u_{-}, & \text {if } x<s t+x_{0} \\ u_{+}, & \text {if } x>s t+x_{0}\end{cases}
$$

where $s=\left(f\left(u_{+}\right)-f\left(u_{-}\right)\right) /\left(u_{+}-u_{-}\right)$is the shock speed.

Theorem 1.3. Let $u_{j}^{n}$ be the solution of (1.1) with initial value (1.14) given by Godunov's scheme. Then the $L^{1}$ norm of the approximation error satisfies

$$
\sum_{j=-\infty}^{\infty}\left|u_{j}^{n}-w(j \Delta x, n \Delta t)\right| \leq\left(6+\frac{\lambda s(1-\lambda s)}{\gamma}\right)\left|u_{+}-u_{-}\right| .
$$

Remark 4. Although our results are for Godunov's scheme, the methods and results are expected to be valid for general strict $l^{1}$-contracting schemes

$$
u_{j}^{n+1}=\left(G\left(u^{n}\right)\right)_{j}=u_{j}^{n}-\lambda\left(F_{j+1 / 2}(u)-F_{j-1 / 2}(u)\right) .
$$


We say a scheme is strictly $l^{1}$-contracting if for any $u-v \in l^{1}$ which is neither nonpositive a.e. nor nonnegative a.e., the inequality

$$
\sum_{j=-\infty}^{\infty}\left|\left(G^{k}(u)\right)_{j}-\left(G^{k}(v)\right)_{j}\right|<\sum_{j=-\infty}^{\infty}\left|u_{j}-v_{j}\right|
$$

holds for sufficiently large $k$.

This paper is divided into four sections after this one. In $\S 2$, we review some well known properties of Godunov's scheme. In $\S 3$, we prove the existence of traveling waves of Godunov's scheme when $\lambda s$ is rational and Theorem 1.2 (in Corollary 3.14). In $\S 4$, we prove the existence result Theorem 1.1. Finally, we show Theorem 1.3 in $\S 5$.

Although the formulation and proofs are for the case where the shock speed $s$ is positive, the proof for negative shock speed is similar and will be omitted. When $s=0$, the existence of the discrete shock and error estimates results hold trivially.

\section{Preliminaries}

In this section, we list some of the known properties of Godunov's scheme.

Lemma 2.1. The Godunov scheme is $l^{1}$-contracting, that is, for any function $u_{j}$ and $v_{j}$ with $u-v \in l^{1}$,

$$
\|G u-G v\|_{l^{1}} \leq\|u-v\|_{l^{1}} .
$$

Corollary 2.2. The operator $T$ is $l^{1}$-contracting, that is, for any function $u_{j}$ and $v_{j}$ with $u-v \in l^{1}$,

$$
\|T u-T v\|_{l^{1}} \leq\|u-v\|_{l^{1}} .
$$

Proof. The conclusion follows immediately from (2.1) and the fact that $T$ is a shift of $G^{m}$.

Lemma 2.3. The Godunov scheme is monotone preserving, that is, if $u$ is decreasing (or increasing), then Gu is also decreasing (or increasing).

Corollary 2.4. The operator $T$ is monotone preserving.

Lemma 2.5. If the function $u_{j}$ is such that $u_{j} \rightarrow u_{ \pm}$as $j \rightarrow \pm \infty$ where $u_{ \pm}$ satisfies

$$
l / m=\lambda\left(f\left(u_{+}\right)-f\left(u_{-}\right)\right) /\left(u_{+}-u_{-}\right),
$$

then

$$
\sum_{j=-\infty}^{\infty}\left[(T u)_{j}-u_{j}\right]=0 .
$$

Proof. From (1.2), we have

$$
\sum_{j=-\infty}^{\infty}\left[u_{j}^{n+1}-u_{j}^{n}\right]=-\lambda\left(f\left(u_{+}\right)-f\left(u_{-}\right)\right)=\lambda s\left(u_{+}-u_{-}\right) .
$$


Then we can prove (2.4) as follows:

$$
\begin{aligned}
& \sum_{j=-\infty}^{\infty}\left[(T u)_{j}-u_{j}\right] \sum_{j=-\infty}^{\infty}\left[u_{j-l}^{m}-u_{j}\right] \\
= & \sum_{j=-\infty}^{\infty}\left[u_{j-l}^{m}-u_{j-l}\right]+\sum_{j=-\infty}^{\infty}\left[u_{j-l}-u_{j}\right] \\
= & \sum_{k=0}^{m-1} \sum_{j=-\infty}^{\infty}\left[u_{j-l}^{k+1}-u_{j}^{k}\right]+l\left(u_{-}-u_{+}\right) \\
= & m \lambda s\left(u_{+}-u_{-}\right)+l\left(u_{-}-u_{+}\right)=0 .
\end{aligned}
$$

\section{EXISTENCE OF TRAVELING WAVES}

In this section, we shall prove the existence of (1.6). We consider $T^{k} U(y)$ where

$$
U_{j}(y)= \begin{cases}u_{-}, & \text {if } j \leq-1, \\ u_{-}+y\left(u_{+}-u_{-}\right), & \text {if } j=0 \\ u_{+}, & \text {if } j \geq 1\end{cases}
$$

and $y \in[0,1]$. We omit $y$ in the following when no ambiguity is expected. Since $\left(T^{k} U\right)_{j}$ depends on $U_{i}, i=j+l, j+l \pm 1, j+l \pm 2, \ldots, j+l \pm k$, we can see that $\left(T^{k} U\right)_{j} \rightarrow u_{ \pm}$as $j \rightarrow \pm \infty$. The following two lemmas are immediate consequences of Corollary 2.4 and Lemma 2.5.

\section{Lemma 3.1.}

$$
\sum_{j=-\infty}^{\infty}\left[\left(T^{k} U\right)_{j}-U_{j}\right]=0
$$

Lemma 3.2. The function $\left(T^{k} U\right)_{j}$ is decreasing.

For a decreasing function $u_{j}$ with $u_{-\infty}>a>u_{\infty}$, there is a number $J(u)$, such that

$$
\bar{f}_{j+1 / 2}(u)= \begin{cases}f\left(u_{j}\right), & \text { if } j \leq J(u), \\ f\left(u_{j+1}\right) \neq f\left(u_{j}\right), & \text { if } j>J(u) .\end{cases}
$$

Lemma 3.3. (i)

$$
\left(T^{k} U\right)_{j} \geq b \quad \text { for } j \leq J\left(T^{k} U\right) .
$$

$$
b>\left(T^{k} U\right)_{J\left(T^{k} U\right)+1} \geq u_{+} .
$$

Proof. Since $T^{k}$ is a shift of $G^{m k}$, it suffices to prove that $G^{k} U$ satisfies the statements (i), (ii) and (iii) with $T^{k}$ replaced by $G^{k} U$, that is to prove

$$
\begin{gathered}
\left(G^{k} U\right)_{j} \geq b \quad \text { for } j \leq J\left(G^{k} U\right), \\
b>\left(G^{k} U\right)_{J\left(G^{k} U\right)+1} \geq u_{+},
\end{gathered}
$$




$$
\left(G^{k} U\right)_{j}=u_{+} \quad \text { for } j \geq J\left(G^{k} U\right)+2,
$$

for $k=0,1,2, \ldots$. For simplicity, we denote $u^{n}=G^{n} U$ in this proof. It is clear that (3.7)-(3.9) hold when $k=0$. Assume, for induction, that (3.7)-(3.9) hold for $k=0, \ldots, n$. We claim that (3.7)-(3.9) are valid for $k=n+1$ as well. To this end, we first observe that

$$
u_{j}^{n+1} \geq b \quad \text { for } j \leq J\left(u^{n}\right) .
$$

Indeed, if $j \leq J\left(u^{n}\right)$, then $b \leq u_{j}^{n} \leq u_{j-1}^{n}$,

$$
u_{j}^{n+1}=u_{j}^{n}-\lambda\left(f\left(u_{j}^{n}\right)-f\left(u_{j-1}^{n}\right)\right) \geq u_{j}^{n} \geq b .
$$

For $j \geq J\left(u^{n}\right)+2$, we have

$$
u_{j}^{n+1}=u_{j}^{n}-\lambda\left(f\left(u_{j+1}^{n}\right)-f\left(u_{j}^{n}\right)\right)=u_{j}^{n}=u_{+} .
$$

If $u_{J\left(u^{n}\right)+1}^{n+1}<b$, then $J\left(u^{n+1}\right)=J\left(u^{n}\right)$ since $f\left(u_{J\left(u^{n}\right)+2}^{n+1}\right)=f\left(u_{+}\right)=f(b)$, and hence (3.7)-(3.9) hold. If $u_{J\left(u^{n}\right)+1}^{n+1} \geq b$, then $f\left(u_{J\left(u^{n}\right)+1}^{n+1}\right) \geq f\left(u_{+}\right)=f\left(u_{J\left(u^{n}\right)+2}^{n+1}\right)$ implies that $J\left(u^{n+1}\right)=J\left(u^{n}\right)+1$ and (3.7)-(3.9) hold. Combining both cases, we see that (3.7)-(3.9) are valid for $k=n+1$ which completes the induction.

\section{Corollary 3.4.}

$$
J\left(G^{n+1} T^{k} U\right) \geq J\left(G^{n} T^{k} U\right)
$$

for any $k \geq 0$ and $n \geq 0$.

Proof. From the proof of Lemma 3.3, we see that

$$
J\left(G^{n+1} U\right) \geq J\left(G^{n} U\right) .
$$

The conclusion then follows from the fact that $T$ is a shift of $G^{m}$.

Lemma 3.5. $\left(T^{k} U\right)_{j=0} \geq b$ for any $k \geq 0$.

Proof. Assume the contrary, i.e $\left(T^{k} U\right)_{0}<b$. Then from Lemma 3.3 (ii), we see that $J\left(T^{k} U\right)<0$. Thus, $\left(T^{k} U\right)_{j}=u_{+}$for $j \geq 1$. This leads to

$$
\begin{aligned}
& 0=\sum_{j=-\infty}^{\infty}\left[\left(T^{k} U\right)_{j}-U_{j}\right], \\
& \sum_{j=-\infty}^{0}\left[\left(T^{k} U\right)_{j}-U_{j}\right] \leq\left(T^{k} U\right)_{0}-u_{-}<0,
\end{aligned}
$$

which is a contradiction.

Lemma 3.6. There are some constants $\alpha>0$ and $\beta>0$ independent of $k \geq 0$ such that

$$
\left|\left(T^{k} U\right)_{j}-u_{-}\right| \leq \alpha \exp (-\beta|j|) \quad \text { for } j \leq 0
$$

and

$$
0 \leq J\left(T^{k} U\right) \leq \frac{\alpha}{\left(1-e^{-\beta}\right)\left(b-u_{+}\right)}
$$


Proof. When $j \leq-l$, the operator $T$ is related to that of the upwinding scheme for which estimate (3.14) was proved in [Je]. For completeness, we present the proof here. Inequality (3.14) holds for any $\alpha, \beta>0$ when $k=0$. Assume, for induction, that (3.14) holds for $k=0,1, \ldots, n$. We claim that (3.14) also holds for $k=n+1$ for some suitable constants $\alpha, \beta>0$ independent of $n$. For simplicity, we denote $u=T^{k} U$ in this proof. From Lemma 3.5, we see that $u_{j} \geq b$ for $j \leq 0$. Then, we have $u_{j}^{i}=\left(G^{i} u\right)_{j} \geq b$ for $j \leq 0$ and any $i \geq 0$. We first consider $u_{j}^{1}-u_{-}$:

$$
\begin{aligned}
& \left|u_{j}^{1}-u_{-}\right|=\left|u_{j}-\lambda\left(f\left(u_{j}\right)-f\left(u_{j-1}\right)\right)\right| \\
& \leq\left(1-\lambda f^{\prime}\left(\theta_{0}\right)\right)\left|u_{j}-u_{-}\right|+\lambda f^{\prime}\left(\theta_{0}\right)\left|u_{j-1}-u_{-}\right| \\
& \leq \alpha\left[\left(1-\lambda f^{\prime}\left(\theta_{0}\right)\right)+\lambda f^{\prime}\left(\theta_{0}\right) e^{-\beta}\right] \exp (-\beta|j|)
\end{aligned}
$$

where $f^{\prime}\left(\theta_{0}\right)=\left(f\left(u_{-}\right)-f(b)\right) /\left(u_{-}-b\right)$ and we used the convexity of $f$. Similarly, for $j \leq-l$, we have

$$
\begin{aligned}
& \left|\left(T^{n+1} U\right)_{j}-u_{-}\right|=\left|u_{j+l}^{m}-u_{-}\right| \\
& \leq \alpha\left[1-\lambda f^{\prime}\left(\theta_{0}\right)+\lambda f^{\prime}\left(\theta_{0}\right) e^{-\beta}\right]^{m} e^{\beta l} \exp (-\beta|j|) .
\end{aligned}
$$

Therefore, (3.14) holds for $j \leq-l$ if we can find a constant $\beta>0$ independent of $n$ such that

$$
g(\beta):=\left[1-\lambda f^{\prime}\left(\theta_{0}\right)+\lambda f^{\prime}\left(\theta_{0}\right) e^{-\beta}\right]^{m} e^{\beta l} \leq 1 .
$$

An easy calculation shows that $g(0)=1$ and $g^{\prime}(0)=\lambda m\left(s-f^{\prime}\left(\theta_{0}\right)\right)<0$. Thus we can choose $\beta>0$ such that (3.18) holds. Notice that so far we have not imposed any resitriction on $\alpha>0$. We can let $\alpha$ be large enough so that (3.14) holds for $-l<j \leq 0$ as well. This completes the proof of (3.14).

To prove (3.15), we start with

$$
\sum_{j=1}^{\infty}\left[\left(T^{k} U\right)_{j}-u_{+}\right]=-\sum_{j=-\infty}^{0}\left[\left(T^{k} U\right)_{j}-u_{-}\right] \leq \frac{\alpha}{1-e^{-\beta}} .
$$

On the other hand, we have

$$
\begin{aligned}
& \sum_{j=1}^{\infty}\left[\left(T^{k} U\right)_{j}-u_{+}\right] \geq \sum_{j=1}^{J\left(T^{k} U\right)}\left[\left(T^{k} U\right)_{j}-u_{+}\right] \\
& \geq\left(J\left(T^{k} U\right)\left[b-u_{+}\right] .\right.
\end{aligned}
$$

Combining (3.20) and (3.19), we prove that

$$
J\left(T^{k} U\right) \leq \frac{\alpha}{\left(1-e^{-\beta}\right)\left(b-u_{+}\right)} .
$$

Corollary 3.7.

$$
\left(T^{k} U\right)_{j}=u_{+} \quad \text { for } j \geq J_{0}:=\frac{\alpha}{\left(1-e^{-\beta}\right)\left(b-u_{+}\right)}+2 .
$$

Lemma 3.8. There exists a subsequence $\left\{k_{n}\right\}_{n=1}^{\infty}$ of $\{k\}$ such that $k_{n} \rightarrow \infty$ and

$$
\left(T^{k_{n}} U\right)_{j} \rightarrow v_{j}, \quad\left(T^{k_{n}+1} U\right)_{j} \rightarrow \bar{v}_{j}
$$

for every $j \in \mathbb{Z}$ and

$$
\sum_{j=-\infty}^{\infty}\left|\left(T^{k_{n}} U\right)_{j}-\left(T^{k_{n}+1} U\right)_{j}\right| \rightarrow \sum_{j=-\infty}^{\infty}\left|v_{j}-\bar{v}_{j}\right|
$$

as $n \rightarrow \infty$. 
Proof. Since $T^{k} U$ are bounded and decreasing, there is a subsequence of $\{k\}$, denoted by $\left\{k_{n}\right\}$, such that $k_{n} \rightarrow \infty$,

$$
\left(T^{k_{n}} U\right)_{j} \rightarrow v_{j}, \quad\left(T^{k_{n}+1} U\right)_{j} \rightarrow \bar{v}_{j}
$$

as $n \rightarrow \infty$ for every $j \in \mathbb{Z}$. It remains to prove (3.23). To this end, we have, for any $N>0$,

$$
\begin{aligned}
& \left|\lim _{n \rightarrow \infty} \sum_{j=-\infty}^{\infty}\right|\left(T^{k_{n}} U\right)_{j}-\left(T^{k_{n}+1} U\right)_{j}\left|-\sum_{j=-N}^{J_{0}}\right| v_{j}-\bar{v}_{j}|| \\
& =\lim _{n \rightarrow \infty}\left|\sum_{j=-\infty}^{\infty}\right|\left(T^{k_{n}} U\right)_{j}-\left(T^{k_{n}+1} U\right)_{j}\left|-\sum_{j=-N}^{J_{0}}\right|\left(T^{k_{n}} U\right)_{j}-\left(T^{k_{n}+1} U\right)_{j}|| \\
& =\lim _{n \rightarrow \infty} \sum_{j=-\infty}^{-N-1}\left|\left(T^{k_{n}} U\right)_{j}-\left(T^{k_{n}+1} U\right)_{j}\right| \\
& \leq \frac{2 \alpha}{1-e^{-\beta}} \exp (-\beta N)
\end{aligned}
$$

where we used Corollary 3.7. Letting $N \rightarrow \infty$ in (3.25), we prove (3.23).

To proceed, we need more detailed information about the $l^{1}$-contractiveness of Godunov's scheme.

Lemma 3.9. Let $u_{j}, \bar{u}_{j}$ be decreasing functions satisfying $u \rightarrow u_{ \pm}, \bar{u}_{j} \rightarrow u_{ \pm}$as $j \rightarrow \pm \infty$ and

$$
\begin{aligned}
& u_{J(u)} \geq b, \quad u_{j}=u_{+}, \quad \text { for } j \geq J(u)+2, \\
& \bar{u}_{J(\bar{u})} \geq b, \quad \bar{u}_{j}=u_{+}, \quad \text { for } j \geq J(\bar{u})+2 .
\end{aligned}
$$

Then, the difference $w=u-\bar{u}$ and $G u-G \bar{u}$ satisfy

$$
\begin{aligned}
& \sum_{j=-\infty}^{\infty}\left|(G u)_{j}-(G \bar{u})_{j}\right| \leq \sum_{j=-\infty}^{\infty}\left|u_{j}-\bar{u}_{j}\right| \\
& -2 \sum_{j \leq \min (J(u), J(\bar{u}))} \chi\left(w_{j} w_{j-1}<0\right) \min \left(\left(1-\lambda f^{\prime}\left(\theta_{j}\right)\right)\left|w_{j}\right|,\left|\lambda f^{\prime}\left(\theta_{j-1}\right) w_{j-1}\right|\right) \\
& -2 \chi(J(u)=J(\bar{u})) \chi\left(w_{J(u)+1} w_{J(u)}<0\right) \min \left(\left|w_{J(u)+1}\right|,\left|\lambda f^{\prime}\left(\theta_{J(u)} w_{J(u)}\right)\right|\right) \\
& -2 \chi(J(u)<J(\bar{u})) \chi\left(w_{J(u)+1} w_{J(u)}<0\right) \\
& \quad \min \left(\left(1+\lambda f^{\prime}\left(\theta_{J(u)+1}\right)\right)\left|w_{J(u)+1}\right|,\left|\lambda f^{\prime}\left(\theta_{J(u)} w_{J(u)}\right)\right|\right) \\
& -2 \chi(J(u)>J(\bar{u})) \chi\left(w_{J(\bar{u})+1} w_{J(\bar{u})}<0\right) \\
& \quad \min \left(\left(1+\lambda f^{\prime}\left(\theta_{J(\bar{u})+1}\right)\right)\left|w_{J(\bar{u})+1}\right|,\left|\lambda f^{\prime}\left(\theta_{J(\bar{u})} w_{J(\bar{u})}\right)\right|\right)
\end{aligned}
$$

where

$$
\chi(A)= \begin{cases}1, & \text { if } A \text { is true }, \\ 0, & \text { if } A \text { is false } .\end{cases}
$$

Proof. Without loss of generality, we assume that $J(u) \leq J(\bar{u})$ in this proof. We consider the difference $w_{j}^{1}:=u_{j}^{1}-\bar{u}_{j}$ : 
Case I. $j \leq J(u) \leq J(\bar{u})$.

In this case, the difference $w_{j}^{1}$ satisfies

$$
w_{j}^{1}=w_{j}-\lambda f^{\prime}\left(\theta_{j}\right) w_{j}+\lambda f^{\prime}\left(\theta_{j-1}\right) w_{j-1}
$$

where $f^{\prime}\left(\theta_{j}\right)=\left(f\left(u_{j}\right)-f\left(\bar{u}_{j}\right)\right) /\left(u_{j}-\bar{u}_{j}\right) \geq f^{\prime}(b)>0$.

Case I(i). If $w_{j} w_{j-1} \geq 0$, then

$$
\left|w_{j}^{1}\right|=\left|w_{j}\right|-\lambda f^{\prime}\left(\theta_{j}\right)\left|w_{j}\right|+\lambda f^{\prime}\left(\theta_{j-1}\right)\left|w_{j-1}\right| .
$$

Case I(ii). If $w_{j} w_{j-1}<0$ and $\left|w_{j}\right|\left(1-\lambda f^{\prime}\left(\theta_{j}\right)\right) \geq\left|\lambda f^{\prime}\left(\theta_{j-1}\right) w_{j-1}\right|$, then

$$
\left|w_{j}^{1}\right|=\left|w_{j}\right|-\lambda f^{\prime}\left(\theta_{j}\right)\left|w_{j}\right|+\lambda f^{\prime}\left(\theta_{j-1}\right)\left|w_{j-1}\right|-2 \lambda f^{\prime}\left(\theta_{j-1}\right)\left|w_{j-1}\right| .
$$

Case I(iii). If $w_{j} w_{j-1}<0$ and $\left|w_{j}\right|\left(1-\lambda f^{\prime}\left(\theta_{j}\right)\right)<\left|\lambda f^{\prime}\left(\theta_{j-1}\right) w_{j-1}\right|$, then

$$
\left|w_{j}^{1}\right|=\left|w_{j}\right|-\lambda f^{\prime}\left(\theta_{j}\right)\left|w_{j}\right|+\lambda f^{\prime}\left(\theta_{j-1}\right)\left|w_{j-1}\right|-2\left(1-\lambda f^{\prime}\left(\theta_{j}\right)\right)\left|w_{j}\right| .
$$

Combining Case I (i)-(iii), we obtain

$$
\begin{aligned}
& \left|w_{j}^{1}\right|=\left|w_{j}\right|-\lambda f^{\prime}\left(\theta_{j}\right)\left|w_{j}\right|+\lambda f^{\prime}\left(\theta_{j-1}\right)\left|w_{j-1}\right| \\
& -2 \chi\left(w_{j} w_{j-1}<0\right) \min \left(\left(1-\lambda f^{\prime}\left(\theta_{j}\right)\right)\left|w_{j}\right|,\left|\lambda f^{\prime}\left(\theta_{j-1}\right) w_{j-1}\right|\right) .
\end{aligned}
$$

Case II. $j=J(u)+1=J(\bar{u})+1$.

For this case, we can prove in the same way as in Case I that

$$
\begin{aligned}
& \left|w_{J(u)+1}^{1}\right|=\left|w_{J(u)+1}\right|+\lambda f^{\prime}\left(\theta_{J(u)}\right)\left|w_{J(u)}\right| \\
& -2 \chi\left(w_{J(u)+1} w_{J(u)}<0\right) \min \left(\left|w_{J(u)+1}\right|, \mid \lambda f^{\prime}\left(\theta_{J(u)} w_{J(u)} \mid\right) .\right.
\end{aligned}
$$

Case III. $j=J(u)+1<J(\bar{u})+1$.

From (3.5), we can see that $u_{J(u)+1}<b$ and $\bar{u}_{J(u)+1} \geq b$ and hence $w_{J(u)+1}<0$. Consider $w_{J(u)+1}^{1}$ :

$$
\begin{aligned}
& \left|w_{J(u)+1}^{1}\right|=\mid w_{J(u)+1}-\lambda f^{\prime}\left(\theta_{J(u)+1}\right) w_{J(u)+1} \\
& \quad-\lambda\left(f\left(u_{J(u)+2}\right)-f\left(u_{J(u)+1}\right)\right)\left|+\lambda f^{\prime}\left(\theta_{J(u)}\right)\right| w_{J(u)} \mid \\
& -2 \chi\left(w_{J(u)+1} w_{J(u)}<0\right) \min \left(\mid w_{J(u)+1}-\lambda f^{\prime}\left(\theta_{J(u)+1}\right) w_{J(u)+1}\right. \\
& \left.\quad-\lambda\left(f\left(u_{J(u)+2}\right)-f\left(u_{J(u)+1}\right)\right)|,| \lambda f^{\prime}\left(\theta_{J(u)}\right) w_{J(u)} \mid\right) .
\end{aligned}
$$

We note that $w_{J(u)+1}$ and $-\lambda\left(f\left(u_{J(u)+2}\right)-f\left(u_{J(u)+1}\right)\right)$ are nonpositive which reduces (3.34) to

$$
\begin{aligned}
& \left|w_{J(u)+1}^{1}\right| \leq\left|w_{J(u)+1}\right|+\lambda\left(f\left(u_{J(u)+2}\right)-f\left(\bar{u}_{J(u)+1}\right)\right)+\lambda f^{\prime}\left(\theta_{J(u)}\right)\left|w_{J(u)}\right| \\
& -2 \chi\left(w_{J(u)+1} w_{J(u)}<0\right) \min \left(\left|w_{J(u)+1}\right|\left(1-\lambda f^{\prime}\left(\theta_{J(u)+1}\right)\right),\left|\lambda f^{\prime}\left(\theta_{J(u)}\right) w_{J(u)}\right|\right) .
\end{aligned}
$$

Case IV. $J(u)+2 \leq j \leq J(\bar{u})$.

In this case, $w_{j}<0$ and $w_{j}^{1}<0$ and hence

$$
\left|w_{j}^{1}\right|=\left|w_{j}\right|+\lambda\left(f\left(u_{j+1}\right)-f\left(\bar{u}_{j}\right)\right)-\lambda\left(f\left(u_{j}\right)-f\left(\bar{u}_{j-1}\right)\right) .
$$

Case $V \cdot j=J(\bar{u})+1>J(u)+1$.

Since both $w_{J(\bar{u})+1}$ and $w_{J(\bar{u})+1}^{1}$ are nonpositive, we have

$$
\left|w_{J(\bar{u})+1}^{1}\right|=\left|w_{J(\bar{u})+1}\right|+\lambda\left(f\left(u_{J(\bar{u})+2}\right)-f\left(\bar{u}_{J(\bar{u})+2}\right)\right)-\lambda\left(f\left(u_{J(\bar{u})+1}\right)-f\left(\bar{u}_{J(\bar{u})}\right)\right) .
$$


Case VI. $j \leq J(\bar{u})+2$.

In this case, $w_{j}^{1}=w_{j}=0$.

Combining Case I-VI, we obtain (3.27).

Theorem 3.10. The function $v_{j}(y)$ obtained in Lemma 3.8 is a family of traveling wave solutions of Godunov's scheme parametrized by $y \in[0,1]$, i.e., $T v(y)=v(y)$. Furthermore, these traveling waves satisfy

$$
\begin{gathered}
\sum_{j=-\infty}^{\infty}\left(v_{j}(y)-U_{j}(y)\right)=0, \\
\left|v_{j}-u_{-}\right| \leq \alpha \exp (-\beta|j-J(v)|) \quad \text { for } j \leq J(v), \\
v_{j} \geq b \quad \text { for } j \leq J(v), \\
v_{j}=u_{+} \quad \text { for } j \geq J(v)+2
\end{gathered}
$$

for some constants $\alpha, \beta>0$ independent of $y \in[0,1]$.

Proof. We observe that $T v=\lim _{n \rightarrow \infty} T\left(T^{k_{n}} U\right)=\bar{v}$. Therefore, the assertion to be proved is equivalent to $v=\bar{v}$. To prove this, we assume

$$
\sum_{-\infty}^{\infty}\left|v_{j}-\bar{v}_{j}\right|=C_{0}>0
$$

a contradiction. Lemma 2.5 implies that $\sum_{-\infty}^{\infty}\left(v_{j}-\bar{v}_{j}\right)=0$. Then there exist $j_{+}, j_{-} \in \mathbb{Z}$ such that $v_{j_{+}}-\bar{v}_{j_{+}}>0$ and $v_{j_{-}}-\bar{v}_{j_{-}}<0$. From Lemma 3.3, we can see that there are grid points $J(v)$ and $J(\bar{v})$,

$$
0 \leq J(v), \quad J(\bar{v}) \leq \frac{\alpha}{\left(1-e^{-\beta}\right)\left(b-u_{+}\right)}
$$

such that

$$
\begin{aligned}
& v_{j} \geq b \quad \text { for } j \leq J(v), \\
& v_{J(v)+1} \in\left[b, u_{+}\right] \\
& v_{j}=u_{+} \quad \text { for } j \geq J(v)+2,
\end{aligned}
$$

and

$$
\begin{aligned}
& \bar{v}_{j} \geq b \quad \text { for } j \leq J(\bar{v}), \\
& \bar{v}_{J(\bar{v})+1} \in\left[b, u_{+}\right] \\
& \bar{v}_{j}=u_{+} \quad \text { for } j \geq J(\bar{v})+2 .
\end{aligned}
$$

Thus, it is clear that

$$
j_{+}, j_{-} \leq \max (J(v), J(\bar{v}))+1 .
$$

We can further choose $j_{+}$and $j_{-}$such that

$$
v_{j}=\bar{v}_{j} \quad \text { for } j \text { between } j_{+} \text {and } j_{-} .
$$

We introduce the following notations:

$$
j_{1}:=\min \left(j_{+}, j_{-}\right), \quad j_{2}:=\max \left(j_{+}, j_{-}\right) .
$$


By Lemma 3.8, for any $\epsilon>0$ and $\eta>0$, there is a $N(\epsilon, \eta)>0$ such that for $n \geq N(\epsilon, \eta)$,

$$
\left\|T^{k_{n}} U-T^{k_{n}+1} U\right\|_{l^{1}} \leq\|v-\bar{v}\|_{l^{1}}+\epsilon,
$$

and

$$
\begin{gathered}
\left|\left(T^{k_{n}} U\right)_{j}-v_{j}\right|<\eta, \\
\left|\left(T^{k_{n}+1} U\right)_{j}-\bar{v}_{j}\right|<\eta,
\end{gathered}
$$

for $j_{1} \leq j \leq j_{2}$.

Case A. $j_{1}=j_{2}-1$.

For simplicity, we use the notation

$$
u:=T^{k_{n}} U, \quad \bar{u}:=T^{k_{n}+1} U
$$

in this proof. By choosing $\eta>0$ small enough, we have

$$
\left(u_{j_{1}}-\bar{u}_{j_{1}}\right)\left(u_{j_{2}}-\bar{u}_{j_{2}}\right)=\left(v_{j_{1}}-\bar{v}_{j_{1}}\right)\left(v_{j_{2}}-\bar{v}_{j_{2}}\right)+O(1) \eta<0 .
$$

By using the $l^{1}$-contractiveness of Godunov's scheme and Lemma 3.9, we obtain

$$
\begin{aligned}
& \|v-\bar{v}\|_{l^{1}} \leq\left\|T^{k_{n+1}-k_{n}}\left(T^{k_{n}} U\right)-T^{k_{n+!}-k_{n}}\left(T^{k_{n}+1} U\right)\right\|_{l^{1}} \\
& \leq\left\|G T^{k_{n}} U-G T^{k_{n}+1} U\right\|_{l^{1}} \\
& \leq\|v-\bar{v}\|_{l^{1}}-|O(1)| \min \left(\left|v_{j_{1}}-\bar{v}_{j_{1}}\right|,\left|v_{j_{2}}-\bar{v}_{j_{2}}\right|\right)
\end{aligned}
$$

which is a contradiction.

Case B. $j_{1}<j_{2}-1$.

In this case, we have $v_{j}=\bar{v}_{j}$ for $j_{1}<j<j_{2}$ and hence $\left|u_{j}-\bar{u}_{j}\right|<2 \eta$. For definiteness, we assume that

$$
v_{j_{1}}-\bar{v}_{j_{1}}>0, \quad v_{j_{2}}-\bar{v}_{j_{2}}<0 .
$$

Since $u_{j}-\bar{u}_{j}$ does not change sign for $j>\min (J(u), J(\bar{u}))+1$, we have

$$
j_{2} \leq \min (J(u), J(\bar{u}))+1 .
$$

There are the following three cases for $j_{2}$.

Case $B(i) . j_{2} \leq \min (J(u), J(\bar{u}))$.

In this case, the difference $u_{j}^{1}-\bar{u}_{j}^{1}$ has the form

$$
w_{j}^{1}=w_{j}\left(1-\lambda f^{\prime}\left(\theta_{j}\right)\right)+\lambda f^{\prime}\left(\theta_{j-1}\right) w_{j-1}
$$

from which we can see that

$$
u_{j_{1}+1}^{1}-\bar{u}_{j_{1}+1}^{1}=\lambda f^{\prime}\left(\theta_{j_{1}}\right)\left(u_{j_{1}}-\bar{u}_{j_{1}}\right)+O(1) \eta=\lambda f^{\prime}\left(\theta_{j_{1}}\right)\left(v_{j_{1}}-\bar{v}_{j_{1}}\right)+O(1) \eta,
$$

$$
\begin{gathered}
u_{j}^{1}-\bar{u}_{j}^{1}=O(1) \eta \\
u_{j_{2}}^{1}-\bar{u}_{j_{2}}^{1}=\left(1-\lambda f^{\prime}\left(\theta_{j_{2}}\right)\left(v_{j_{2}}-\bar{v}_{j_{2}}\right)+O(1) \eta .\right.
\end{gathered}
$$

Similarly, we can show that

$$
u_{j_{2}-1}^{j_{2}-j_{1}-1}-\bar{u}_{j_{2}-1}^{j_{2}-j_{1}-1}=|O(1)|\left(v_{j_{1}}-\bar{v}_{j_{1}}\right)+O(1) \eta>0,
$$




$$
u_{j_{2}}^{j_{2}-j_{1}-1}-\bar{u}_{j_{2}}^{j_{2}-j_{1}-1}=|O(1)|\left(v_{j_{2}}-\bar{v}_{j_{2}}\right)+O(1) \eta<0
$$

where all constants $O(1)$ are independent of $\epsilon>0$ and $\eta>0$. By applying arguments used in Case A to $u^{j_{2}-j_{1}-1}$ and $\bar{u}^{j_{2}-j_{1}-1}$, we can prove that this case is impossible to occur.

Case B(ii). $j_{2}=\min (J(u), J(\bar{u}))+1$ and $J(u) \neq J(\bar{u})$.

For large $n$, the fact that $v_{j_{2}}-\bar{v}_{j_{2}}<0$ implies that $u_{j_{2}}-\bar{u}_{j_{2}}<0$. This together with $u_{J(u)} \geq b$ and $\bar{u}_{J(\bar{u})+1} \geq b$ infer that $J(u) \leq J(\bar{u})$ and hence $j_{2}=J(u)+1<$ $J(\bar{u})+1$. We estimate the difference $w=u-\bar{u}$ as follows

$$
\begin{aligned}
& w_{j_{2}}^{1}=w_{j_{2}}-\lambda\left(\max \left(f\left(u_{j_{2}+1}\right), f\left(u_{j_{2}}\right)\right)-f\left(u_{j_{2}-1}\right)\right)+\lambda\left(f\left(\bar{u}_{j_{2}}\right)-f\left(\bar{u}_{j_{2}-1}\right)\right) \\
& \leq w_{j_{2}}-\lambda\left(f\left(u_{j_{2}}\right)\right)-f\left(u_{j_{2}-1}\right)+\lambda\left(f\left(\bar{u}_{j_{2}}\right)-f\left(\bar{u}_{j_{2}-1}\right)\right) \\
& =w_{j_{2}}\left(1-\lambda f^{\prime}\left(\theta_{j_{2}}\right)\right)+O(1) \eta<0
\end{aligned}
$$

$$
\begin{gathered}
w_{j}^{1}=O(1) \eta, \quad \text { for } j_{1}<j<j_{2}, \\
w_{j_{1}+1}^{1}=|O(1)|\left(v_{j_{1}}-\bar{v}_{j_{1}}\right)+O(1) \eta .
\end{gathered}
$$

From these calculations, we can see that

$$
\begin{aligned}
& w_{j_{2}-1}^{j_{2}-j_{1}-1}=|O(1)|\left(v_{j_{1}}-\bar{v}_{j_{1}}\right)+O(1) \eta \\
& w_{j_{2}}^{j_{2}-j_{1}-1}=|O(1)|\left(v_{j_{2}}-\bar{v}_{j_{2}}\right)+O(1) \eta .
\end{aligned}
$$

Now we can apply the arguments for Case A to show that this case is also impossible to occur.

Case B(iii). $j_{2}=J(u)+1=J(\bar{u})+1$.

Suppose that $j_{2}=J\left(u^{i}\right)+1=J\left(\bar{u}^{i}\right)+1$ for $i=0,1, \ldots, K$ and $J\left(u^{K+1}\right) \neq$ $J\left(\bar{u}^{K+1}\right)$. Then as long as $j_{1}+i+1<j_{2}$, the function $w^{i+1}$ satisfies

$$
\begin{gathered}
w_{j_{1}+i+1}^{i+1}=|O(1)|\left(v_{j_{1}}-\bar{v}_{j_{1}}\right)+O(1) \eta, \\
w_{j}^{i+1}=O(1) \eta, \quad \text { for } j_{1}+i<j<j_{2}, \\
w_{j_{2}}^{i+1}=|O(1)|\left(v_{j_{2}}-\bar{v}_{j_{2}}\right)+O(1) \eta,
\end{gathered}
$$

If $j_{2}-j_{1}-1 \leq K+1$, then we can take $i=j_{2}-j_{1}-2$ in (3.54) to obtain

$$
\begin{aligned}
& w_{j_{2}-1}^{j_{2}-j_{1}-1}=|O(1)|\left(v_{j_{1}}-\bar{v}_{j_{1}}\right)+O(1) \eta, \\
& w_{j_{2}}^{j_{2}-j_{1}-1}=|O(1)|\left(v_{j_{2}}-\bar{v}_{j_{2}}\right)+O(1) \eta,
\end{aligned}
$$

which, together with the argument for Case A, implies a contradiction.

If $j_{2}-j_{1}-1>K+1$, then there are two possibilities: The first possibility is that $j_{2} \leq \min \left(J\left(u^{K+1}\right), J\left(\bar{u}^{K+1}\right)\right)$. The argument for Case B(i) can be applied to $u^{K+1}$ and $\bar{u}^{K+1}$ to yield a contradiction. The second possibility is that $j_{2}=$ $\min \left(J\left(u^{K+1}\right), J\left(\bar{u}^{K+1}\right)\right)+1$. Then the fact that $w_{j_{2}}^{K+1}=u_{j_{2}}^{K+1}-\bar{u}_{j_{2}}^{K+1}<0,(3.54 \mathrm{c})$, implies that $j_{2}=J(u)+1<J(\bar{u})+1$ because otherwise, one would get

$$
0>u_{j_{2}}^{K+1}-\bar{u}_{j_{2}}^{K+1} \geq b-\bar{u}_{J(\bar{u})+1}^{K+1}>0
$$


which is a contradiction. We can then apply the argument for Case B(ii) to prove that $j_{2}=J(u)+1<J(\bar{u})+1$ also leads to a contradiction. These contradictions show that Case B(iii) cannot occur.

Since both Cases A and B which include all possibilities lead to contradictions, it is necessary that $v=\bar{v}$. Estimates $(3.37 \mathrm{~b}, \mathrm{c}, \mathrm{d})$ follow immediately from Lemma 3.3 and Lemma 3.6.

Corollary 3.11. Let $\phi_{j}$ and $\psi_{j}$ be two traveling wave solutions, in the sense of (1.6), of the same speed, $\lambda s=l / m$, of Godunov's scheme. Then either $\phi_{j} \geq \psi_{j}$ for all $j$ or $\phi_{j} \leq \psi_{j}$ for all $j$.

Proof. The proof of (i) is already contained in that of Theorem 3.10.

Corollary 3.12. If $u_{j}-v_{j}$ is neither nonpositive a.e nor nonnegative a.e and $\sum_{j=-\infty}^{\infty}\left|u_{j}-v_{j}\right|<\infty$, then

$$
\sum_{j=-\infty}^{\infty}\left|\left(G^{n} u\right)_{j}-\left(G^{n} v\right)_{j}\right|<\sum_{j=-\infty}^{\infty}\left|u_{j}-v_{j}\right|
$$

for some $n \in \mathbb{Z}_{+}$.

The proof is very similar to that of Lemma 3.9 and Theorem 3.10. Since we will not use this corollary in this paper, we omit its proof.

Let $v_{j}(y)$ be the family of traveling waves of Godunov's scheme obtained in Theorem 3.10. For any $x \in \mathbb{R}$ can be uniquely written as $x=j+y$ where $j \in \mathbb{N}$ and $y \in[0,1)$. We define, at $x=j+y, j \in \mathbb{N}, y \in[0,1)$,

$$
u(x)=v_{j}(y) .
$$

Theorem 3.13. Assume that $\lambda s$ is rational. There is a unique, up to a shift, Lipschitz continuous traveling wave solution, $u(x), x \in \mathbb{R}$, of Godunov's scheme in the sense of (1.5). Furthermore, this traveling wave solution is decreasing and satisfies

$$
\begin{gathered}
\left|u(x)-u_{-}\right| \leq \alpha \exp (-\beta|x-J(u)|) \quad \text { for } x \leq J(u), \\
u(x) \geq b \quad \text { for } x \leq J(u), \\
u(x)=u_{+} \quad \text { for } x \geq J(v)+2,
\end{gathered}
$$

for some constants $\alpha, \beta>0$. Moreover, the Lipschitz constant of $u(x)$ is $\left|u_{-}-u_{+}\right|$.

Proof. We claim that the function $u(x)$ defined in (3.56) is the traveling wave solution. It is clear from the construction of $u(x)$ that for any fixed $x, u(x+j)$ is a traveling wave solution in the sense of (1.6).

We first prove that $u(x)$ is decreasing and Lipschitz continuous. Given any $x_{1}<x_{2}$, we decompose them as $x_{1}=j_{1}+y_{1}$ and $x_{2}=j_{2}+y_{2}$ where $j_{1}, j_{2} \in \mathbb{Z}$ and $y_{1}, y_{2} \in[0,1)$. Let $v_{j}(y)$ be the traveling wave solution given in Theorem 3.10. Then functions $v_{j+j_{1}}\left(y_{1}\right)=u\left(j+x_{1}\right)$ and $u\left(j+x_{2}\right)$ are also traveling wave solutions 
in the sense of (1.6) which satisfies

$$
\begin{aligned}
& \sum_{j=-\infty}^{\infty}\left(u\left(j+x_{2}\right)-u\left(j+x_{1}\right)\right) \\
& =\sum_{j=-\infty}^{\infty}\left(v_{j+j_{2}}\left(y_{2}\right)-v\left(y_{2}\right)\right)+\sum_{j=-\infty}^{\infty}\left(v\left(y_{2}\right)-v\left(y_{1}\right)\right) \\
& +\sum_{j=-\infty}^{\infty}\left(v\left(y_{1}\right)-v_{j+j_{1}}\left(y_{1}\right)\right)=\left(u_{+}-u_{-}\right)\left(x_{2}-x_{1}\right)<0 .
\end{aligned}
$$

Then Corollary 3.11 and (3.58) imply the monotonicity and Lipschitz continuity:

$$
0 \geq u\left(x_{2}\right)-u\left(x_{1}\right)>\left(u_{+}-u_{-}\right)\left(x_{2}-x_{1}\right) .
$$

Now, we prove that $u(x)$ satisfies traveling wave equation (1.5). To this end, we notice that $u(j+x-\lambda s)$ and $G u(j+x)$ are traveling waves in the sense of (1.6). Then Corollary 3.11 and

$$
\sum_{j=-\infty}^{\infty}(u(j+x-\lambda s)-G u(j+x))=0
$$

imply $u(j+x-\lambda s)-G u(j+x)=0$ for all $j \in \mathbb{Z}$ and hence $u(x-\lambda s)=G u(x)$. To prove equation $(1.5)_{2}$, we observe that

$$
U_{j}(y)=\int_{j-1}^{j} \bar{U}(x+y) d x
$$

where

$$
\bar{U}(x):= \begin{cases}u_{-}, & \text {if } x<0, \\ u_{+}, & \text {if } x>0 .\end{cases}
$$

Using the definition of integral and (3.37a), we obtain

$$
\begin{aligned}
& \sum_{j=-\infty}^{\infty}\left[u(j+y)-\int_{j-1 / 2}^{j+1 / 2} u(x+y) d x\right] \\
= & \sum_{j=-\infty}^{\infty}\left[U_{j}(y)-\int_{j-1 / 2}^{j+1 / 2} U(x+y) d x\right] \\
= & \sum_{j=-\infty}^{\infty}\left[\int_{j-1}^{j} \bar{U}(x+y) d x-\int_{j-1 / 2}^{j+1 / 2} U(x+y) d x\right]=0 .
\end{aligned}
$$

The function $u(x)$ obviously satisfies $(1.5)_{3}$.

Suppose there is another continuous traveling wave in the sense of (1.5), denoted by $u_{1}(x)$. Then, there is a number $x_{0} \in \mathbb{R}$ such that

$$
\int_{\mathbb{R}}\left(u_{1}\left(x+x_{0}\right)-u(x)\right) d x=0 .
$$

We claim that $u_{1}\left(x+x_{0}\right)=u(x)$. Indeed, if otherwise, there would be a point $x_{1}$ such that $u_{1}\left(x_{1}+x_{0}\right)>u\left(x_{1}\right)$. Both $u_{1}\left(x_{1}+x_{0}+j\right)$ and $u\left(x_{1}+j\right)$ are traveling wave solutions in the sense of (1.6). By Corollary 3.11, we have $u_{1}\left(x_{1}+x_{0}+j\right) \geq u\left(x_{1}+j\right)$ for all $j \in \mathbb{Z}$. This, however, contradicts equation (1.5) 2 and (3.62), which proves the uniqueness of the solution of (1.5). 
Estimates (3.57) follow immediately from (3.37) and the fact that $u(x)$ is decreasing.

Corollary 3.14. Let $u(x)$ be the solution of (1.5). Then any solution $v_{j}$ of (1.6) can be written as $v_{j}=u\left(j+x_{0}\right)$ for some $x_{0} \in \mathbb{R}$ independent of $j \in \mathbb{Z}$.

Proof. There is an $x_{0} \in \mathbb{R}$ such that

$$
\sum_{j=-\infty}^{\infty}\left(v_{j}-u\left(j+x_{0}\right)\right)=0
$$

since

$$
\sum_{j=-\infty}^{\infty}\left(u\left(j+x_{0}\right)-\int_{j-1 / 2}^{j+1 / 2} u\left(x+x_{0}\right)\right) d x
$$

and

$$
\int_{\mathbb{R}}\left(u\left(x+x_{0}\right)-\bar{U}(x)\right) d x
$$

is continuous in $x_{0}$ and $\rightarrow \pm \infty$ as $x_{0} \rightarrow \pm \infty$. The functions $u\left(x_{0}+j\right)$ and $v_{j}$ are solutions of (1.6). Then Corollary 3.11 and (3.63) imply that $v_{j}=u\left(x_{0}+j\right)$ for all $j \in \mathbb{Z}$.

\section{Existence of traveling WAVES FOR Godunov'S SCHEME WITH IRRATIONAL $\lambda s$}

In this section, we shall extend our results on the existence of traveling waves of Godunov's scheme to the case where $\lambda s$ is irrational. We shall prove the existence of such traveling waves by taking the limit of a sequence of traveling waves with rational $\lambda s$. For this purpose, we need more information on traveling waves with rational $\lambda s$

Lemma 4.1. Let $\lambda s=l / m$.

(i) There is a traveling wave solution, $v$, in the sense of (1.6), of Godunov's scheme that satisfies $(3.37 b, c, d)$ and

$$
\sum_{j=-\infty}^{\infty}\left|v_{j}-V_{j}\right| \leq\left|u_{+}-u_{-}\right|+\frac{\lambda s}{2 \alpha}(\lambda s-1)\left(u_{+}-u_{-}\right)
$$

where

$$
V_{j}= \begin{cases}u_{-}, & \text {if } j \leq J(v), \\ u_{+}, & \text {if } j>J(v) .\end{cases}
$$

(ii) Any traveling wave solutions, $v_{j}$, in the sense of (1.6), of Godunov's scheme satisfiy (3.37) for some constant $\alpha>0$ and $\beta>0$ and

$$
\sum_{j=-\infty}^{\infty}\left|v_{j}-V_{j}\right| \leq\left(2+\frac{\lambda s(1-\lambda s)}{2 \gamma}\right)\left|u_{+}-u_{-}\right| .
$$

(iii) The continuous traveling wave solution, $u(x)$, in the sense of (1.5), satisfies

$$
\int_{\mathbb{R}}|u(x)-V(x)| d x \leq\left(3+\frac{\lambda s(1-\lambda s)}{2 \gamma}\right)\left|u_{+}-u_{-}\right|
$$


where

$$
V(x)= \begin{cases}u_{-}, & \text {if } x \leq J(u)+1 \\ u_{+}, & \text {if } x>J(u)+1\end{cases}
$$

Proof. (i) We recall the traveling wave equation

$$
v_{j-l}=v_{j}-\lambda \sum_{k=0}^{m-1}\left(\bar{f}_{j+1 / 2}\left(v^{k}\right)-\bar{f}_{j-1 / 2}\left(v^{k}\right)\right) .
$$

Taking the summation $\sum_{J=-N+l+1}^{\infty} \sum_{j=J}^{\infty}$ on (1.6), we obtain

$$
\begin{aligned}
0 & =\sum_{J=-N+1}^{\infty}\left[\sum_{j=J}^{J+l-1}\left(u_{+}-v_{j}\right)-\lambda \sum_{k=0}^{m-1}\left(f_{+}-\bar{f}_{j+l-1 / 2}^{k}\right)\right] \\
& =\sum_{j=-N+1}^{-N+l-1}(j+N)\left(u_{+}-v_{j}\right) \\
& +\sum_{j=-N+1}^{\infty}\left[l\left(u_{+}-v_{j+l-1}\right)-\lambda \sum_{k=0}^{m-1}\left(f_{+}-\bar{f}_{j+l-1 / 2}^{k}\right)\right] .
\end{aligned}
$$

Letting $N \rightarrow \infty$ in (4.7), we have

$$
0=\frac{l(l-1)}{2}\left(u_{+}-u_{-}\right)+\sum_{j=-\infty}^{\infty}\left[l\left(u_{+}-v_{j+l-1}\right)-\lambda \sum_{k=0}^{m-1}\left(f_{+}-\bar{f}\left(v_{j+l-1}^{k}\right)\right)\right]
$$

We note that

$$
\begin{aligned}
& \sum_{j=-\infty}^{\infty}\left(v_{j}^{k}-v_{j}\right)=-\lambda \sum_{i=0}^{k-1} \sum_{j=-\infty}^{\infty}\left[\bar{f}_{j+1 / 2}^{i}-\bar{f}_{j-1 / 2}^{i}\right] \\
& =-\lambda \sum_{i=0}^{k-1}\left[\bar{f}_{\infty}^{i}-\bar{f}_{-\infty}^{i}\right]=-\lambda k\left(f_{+}-f_{-}\right)
\end{aligned}
$$

Plunging (4.8) into (4.7) and using the Rankine-Hugoniot condition, we obtain

$$
\begin{aligned}
0 & =\frac{l}{2}(\lambda s-1)\left(u_{+}-u_{-}\right)+\sum_{k=0}^{m-1} \sum_{j=-\infty}^{J\left(v^{k}\right)}\left[\frac{l}{m}\left(u_{-}-v_{j}^{k}\right)-\lambda\left(f_{-}-f\left(v_{j}^{k}\right)\right)\right] \\
+ & \sum_{k=0}^{m-1} \sum_{j=J\left(v^{k}\right)+1}^{\infty}\left[\frac{l}{m}\left(u_{+}-v_{j+1}^{k}\right)-\lambda\left(f_{+}-f\left(v_{j+1}^{k}\right)\right)\right]+\sum_{k=0}^{m-1} \frac{l}{m}\left(u_{+}-u_{J\left(v^{k}\right)+1}\right) .
\end{aligned}
$$

We introduce the notation

$$
\gamma:=\min \left(s-\frac{f_{+}-f(a)}{u_{+}-a},-s+\frac{f_{-}-f(a)}{u_{-}-a}\right)>0
$$


from which (4.10) becomes

$$
\sum_{k=0}^{m-1} \sum_{j=-\infty}^{J\left(v^{k}\right)}\left|u_{-}-v_{j}^{k}\right|+\sum_{k=0}^{m-1} \sum_{j=J\left(v^{k}\right)+2}^{\infty}\left|u_{+}-v_{j}^{k}\right| \leq \frac{l}{2 \gamma}(1-\lambda s)\left|u_{-}-u_{+}\right| .
$$

Then, there is at least one of $k=0,1, \ldots, m-1$ such that

$$
\sum_{j=-\infty}^{J\left(v^{k}\right)}\left|u_{-}-v_{j}^{k}\right|+\sum_{j=J\left(v^{k}\right)+2}^{\infty}\left|u_{+}-v_{j}^{k}\right| \leq \frac{l}{m \gamma}(1-\lambda s)\left|u_{-}-u_{+}\right| .
$$

Now, we let $v$ to be the traveling wave solution provided by Theorem 3.10. Then $(3.37 \mathrm{c}, \mathrm{d})$ hold for $v^{k}$. From the proof of Lemma 3.6, we see that (3.37b) also holds for $v^{k}$. The function $v^{k}$ is a traveling wave solution that satisfies $(4.1)$ and $(3.37 \mathrm{~b}$, c, d).

(ii) Let $\phi_{j}$ be a traveling wave solution provided by Theorem 4.2 with the same speed as that of $v_{j}$ that satisfies (3.37) and

$$
\sum_{j=-\infty}^{\infty}\left|\phi_{j}-\bar{V}_{j}\right| \leq\left(1+\frac{\lambda s(1-\lambda s)}{2 \alpha}\right)\left|u_{+}-u_{-}\right|
$$

where

$$
\bar{V}_{j}= \begin{cases}u_{-}, & \text {if } j \leq J(\phi), \\ u_{+}, & \text {if } j \geq \bar{J}(\phi)+1 .\end{cases}
$$

By shifting if necessary, we can assume that $J(\phi)=J(v)$ and hence $\bar{V}_{j}=V_{j}$. Since $\phi_{J(v)+1}<v_{J(v)}$, Lemma 5.1 implies that $\phi_{j+1} \leq v_{j}$ for all $j$, From (3.37b), we have

$$
\left|v_{j}-u_{-}\right| \leq\left|\phi_{j+1}-u_{-}\right| \leq \alpha \exp (-\beta|j-J(v)|) .
$$

This proves that $v$ satisfies $(3.37 \mathrm{~b}, \mathrm{c})$ for some constants $\alpha, \beta>0$. We claim that any traveling wave of Godunov's scheme satisfies $v_{J(v)+3}=u_{+}$. Indeed, if otherwise, i.e. $v_{J(v)+3}>u_{+}$, then $v_{j+1}-\phi_{j}$ are positive at $j=J(v)+2$ and negative at $j=J(v)$ which is impossible in view of Corollary 3.11. From the above observation, we consider $G^{n} v$ to see that for $j \geq J(v)+3, v_{j}^{n}>u_{+}$if and only if $v_{j-1}^{n-1}>b$. In other words, $(4.11 \mathrm{~b}, \mathrm{c})$ holds for $v^{n}$ for large $n$ and hence holds for $v$ itself since it is a traveling wave.

By Corollary 3.11, either $v_{j} \geq \phi_{j}$ for all $j$ or $v_{j} \leq \phi_{j}$ for all $j$ holds. If $v_{j} \geq \phi_{j}$ for all $j$, then $v_{j}>a$ and $\phi_{j}>a$ for $j \leq J(v)$ and $v_{j}=\phi_{j}=u_{+}<a$ for $j \geq J(v)+2$ which yields

$$
\begin{aligned}
& \sum_{j=-\infty}^{\infty}\left|v_{j}-V_{j}\right| \\
& =\sum_{j \leq J(v)}\left|v_{j}-u_{-}\right|+\left|v_{J(v)+1}-u_{+}\right| \\
& \leq\left(1+\frac{\lambda s(1-\lambda s)}{2 \gamma}\right)\left|u_{+}-u_{-}\right|+\left|u_{+}-u_{-}\right| .
\end{aligned}
$$


If $v_{j} \leq \phi_{j}$, then $v_{j} \geq \phi_{j+1}$ since $v_{J(v)} \geq b>\phi_{J(v)+1}$. Then we have

$$
\begin{aligned}
& \sum_{j=-\infty}^{\infty}\left|v_{j}-V_{j}\right| \\
= & \sum_{j \leq J(v)-1}\left|v_{j}-u_{-}\right|+\left|v_{J(v)}-u_{-}\right|+\left|v_{J(v)+1}-u_{+}\right| \\
\leq & \sum_{j \leq J(v)-1}\left|\phi_{j+1}-u_{-}\right|+\left|\phi_{J(v)+1}-u_{+}\right|+\left|v_{J(v)}-u_{-}\right| \\
\leq & \left(1+\frac{\lambda s(1-\lambda s)}{2 \gamma}\right)\left|u_{+}-u_{-}\right|+\left|u_{+}-u_{-}\right| .
\end{aligned}
$$

Combining (4.15) we arrive at (4.3).

(iii) From Theorem 3.13, we can see that $J(u(x+y))=J(u(x))$ for $y \in[0,1)$. Then we have

$$
\begin{aligned}
& \sum_{j=-\infty}^{\infty} \operatorname{sign}(j-J(u)-)\left(u(y+j)-V_{j}\right) \\
& \geq \int_{\mathbb{R}}(u(x)-V(x)) d x-\left|u_{+}-u_{-}\right|
\end{aligned}
$$

which, together with statement (ii), yields the desired result.

Theorem 4.2. If $f$ is convex and $u_{-} \geq u_{+}$, then there is a unique traveling wave solution $u(x)$, in the sense of (1.5), of Godunov's scheme which satisfies

$$
\begin{gathered}
\int_{\mathbb{R}}|u(x)-V(x)| \leq\left(3+\frac{\lambda s(1-\lambda s)}{2 \gamma}\right)\left|u_{+}-u_{-}\right|, \\
u(x) \geq b \quad \text { for } x \leq J(u), \\
u(x)=u_{+} \quad \text { for } x \geq J(u)+2,
\end{gathered}
$$

for some $J(u) \in \mathbb{R}$ and

$$
\left|u(x)-u_{-}\right| \leq \alpha \exp (-\beta|x-J(u)|) \quad \text { for } x<J(u)
$$

for some constant $\alpha, \beta>0$ depending on $\lambda s$ and $u_{ \pm}$. Moreover, the solution is Lipschitz continuous with Lipschitz constant $\left|u_{-}-u_{+}\right|$.

Proof. In view of Theorem 3.13 and Lemma 4.1, it remains to prove the assertions when $\lambda s$ is irrational. To this end, we select a sequence $\left\{u_{-, n}\right\}_{n=1}^{\infty}$ such that $u_{-, n} \rightarrow u_{-}$as $n \rightarrow \infty$ and $\lambda s_{n}:=\lambda\left(f_{+}-f\left(u_{-, n}\right)\right) /\left(u_{+}-u_{-, n}\right)=l_{n} / m_{n}$ are rational. Then, from Theorem 3.13 and (iii) of Lemma 4.1, there is a traveling wave solution $u(x, n)$ connecting $u_{-, n}$ and $u_{+, n}$ which satisfies

$$
\int_{\mathbb{R}}|u(x, n)-V(x)| \leq\left(3+\frac{\lambda s_{n}\left(1-\lambda s_{n}\right)}{2 \gamma_{n}}\right)\left|u_{+, n}-u_{-, n}\right| .
$$

Note that $V(x)$ in (4.17) is independent of $n$, which can be achieved by shifting $u(x, n)$ if necessary. Furthermore, these traveling waves $u(x, n)$ are Lipschitz continuous with Lipschitz constant $<\left|u_{+}-u_{-}\right|+1$ for large $n$ and satisfy $(4.16 \mathrm{~b}, \mathrm{c})$. Thus, there is a subsequence of $\{u(., n)\}$, denoted by $\{u(., n)\}$ again, such that

$$
u(x, n) \rightarrow u(x), \quad \text { a.e. as } n \rightarrow \infty .
$$


From the uniform Lipschitz continuity of $u(., n)$ and $(4.17)$, it is clear that $u(x)$ is Lipschitz continuous and satisfies the traveling wave equation (1.5) and (4.16a, b, c).

From the construction of $u(x)$, we see that

$$
u(x)=\lim _{k \rightarrow \infty}\left(T^{n_{k}} U(x-[x])\right)_{[x]}
$$

for some subsequence $\left\{n_{k}\right\}$ of $\{n\}$. Let $S_{-\lambda s}: v(x) \rightarrow v(x-\lambda s)$ be the shift operator. Then $T=\left(S_{-\lambda s} G\right)^{m}$ and hence

$$
u(x)=\lim _{k \rightarrow \infty}\left(\left(S_{-\lambda s} G\right)^{m n_{k}} U(x-[x])\right)_{[x]} .
$$

Applying the argument used in the proof of (3.14), we can see that (4.16d) holds for some constants $\alpha, \beta>0$ which depend continuously on $l / m$ and $u_{ \pm}$. Thus, estimate (4.16d) is preserved when passing the limit $u_{-, n} \rightarrow u_{-}$and hence (4.16d) holds for irrational $\lambda s$ as well.

The proof of the uniqueness of solution of (1.5) when $\lambda s$ is irrational is similar to that of Theorem 3.13 except that we do not have the help from Corollary 3.11. Suppose there is another continuous traveling wave in the sense of (1.5), denoted by $u_{1}(x)$. Then, there is a number $x_{0} \in \mathbb{R}$ such that

$$
\int_{\mathbb{R}}\left(u_{1}\left(x+x_{0}\right)-u(x)\right) d x=0 .
$$

We claim that $u_{1}\left(x+x_{0}\right)=u(x)$. To this end, we let $x_{1}$ be such that

$$
\sum_{j=-\infty}^{\infty}\left|u_{1}\left(x_{1}+x_{0}+j\right)-u\left(x_{1}+j\right)\right|=\min _{x \in[0,1]} \sum_{j=-\infty}^{\infty}\left|u_{1}\left(x+x_{0}+j\right)-u(x+j)\right| .
$$

The equation $(1.5)_{2}$ and (4.19) yield

$$
\sum_{j=-\infty}^{\infty}\left[u_{1}\left(x_{1}+x_{0}+j\right)-u\left(x_{1}+j\right)\right]=\int_{-\infty}^{\infty}\left[u_{1}\left(x_{1}+x_{0}+x\right)-u\left(x_{1}+x\right)\right] d x=0 .
$$

If $u_{1}\left(x_{1}+x_{0}+j_{0}\right)>u\left(x_{1}+j_{0}\right)$ for some $j_{0} \in \mathbb{Z}$, then (4.21) implies that there is a $j_{1} \in \mathbb{Z}$ such that $u_{1}\left(x_{1}+x_{0}+j_{1}\right)<u\left(x_{1}+j_{1}\right)$. Then, Lemma 3.9 infers that

$$
\sum_{-\infty}^{\infty}\left|u_{1}\left(x_{1}+x_{0}+j-\lambda s n\right)-u\left(x_{1}+j-\lambda s n\right)\right|<\sum_{-\infty}^{\infty}\left|u_{1}\left(x_{1}+x_{0}+j\right)-u\left(x_{1}+j\right)\right|
$$

which violates the definition of $x_{0}$. Thus, we have

$$
u_{1}\left(x_{1}+x_{0}+j\right)=u\left(x_{1}+j\right)
$$

for all $j \in \mathbb{Z}$ and hence

$$
u_{1}\left(x_{1}+x_{0}+j-\lambda s n\right)=u\left(x_{1}+j-\lambda s n\right) .
$$

Since $\lambda s$ is irrational, the set $\{j-\lambda s n: j \in \mathbb{Z}, n \in \mathbb{N}\}$ is dense in $\mathbb{R}$. Then the continuity of traveling waves $u_{1}$ and $u$ and (4.22) yield the uniqueness desired. 


\section{ERROR ESTIMATES FOR SINGLE SHOCK SOLUTIONS}

In this section, we shall prove that the $L^{1}$ norm of the approximation error of Godunov's scheme on single shock solutions is of $\Delta x$ order.

Now, we consider the initial value problem

$$
\begin{aligned}
& u_{t}+f(u)_{x}=0, \\
& u(x, 0)= \begin{cases}u_{-}, & \text {if } x<x_{0}, \\
u_{+}, & \text {if } x>x_{0},\end{cases}
\end{aligned}
$$

where $u_{-}>u_{+}$. The solution of (5.1) is the shock

$$
w(x, t)= \begin{cases}u_{-}, & \text {if } x<s t+x_{0}, \\ u_{+}, & \text {if } x>s t+x_{0}\end{cases}
$$

where $s=\left(f\left(u_{+}\right)-f\left(u_{-}\right)\right) /\left(u_{+}-u_{-}\right)$is the shock speed. We are concerned with the accuracy of the numerical solution, $u_{j}^{n}$, given by Godunov's scheme for the initial value problem (5.1).

Theorem 5.3. Let $u_{j}^{n}$ be the solution of (5.1) given by Godunov's scheme. Then the $L^{1}$ norm of the approximation error satisfies

$$
\left\|u_{x}^{n}-w(x, n \Delta t)\right\|_{L^{1}}=\left(6+\frac{\lambda s(1-\lambda s)}{\gamma}\right)\left|u_{+}-u_{-}\right| \Delta x .
$$

Proof. Without loss of generality, we assume that $x_{0}=0$ in (5.1) and (5.2). Let $\phi_{x}$ be a traveling wave solution provided by Theorem 4.2 with $\phi_{j} \rightarrow u_{ \pm}$as $j \rightarrow \pm \infty$ and

$$
\sum_{j=-\infty}^{\infty}\left|\phi_{j}-V_{j}\right| \leq\left(1+\frac{\lambda s(1-\lambda s)}{2 \alpha}\right)\left|u_{+}-u_{-}\right|
$$

We define

$$
w_{j}^{n}:=\frac{1}{\Delta x} \int_{(j-1 / 2) \Delta x}^{(j+1 / 2) \Delta x} w(x, n \Delta t) .
$$

We can see from (5.2) that

$$
w_{j}^{n}= \begin{cases}u_{-}, & \text {if } j<K, \\ w_{K}^{n}, & \text { if } j=K, \\ u_{+}, & \text {if } j>K,\end{cases}
$$

where $K=\left[s t+x_{0}\right]$ or $\left[s t+x_{0}\right]+1$.

There is a $j_{0} \in \mathbb{Z}$ such that

$$
\sum_{j=-\infty}^{\infty}\left|\phi_{j+j_{0}}-w_{j}^{0}\right|=\min _{i \in \mathbb{Z}} \sum_{j=-\infty}^{\infty}\left|\phi_{j+i}-w_{j}^{0}\right|:=C_{0} .
$$


To estimate $C_{0}$, we notice that

$$
\begin{aligned}
& \sum_{j=-\infty}^{\infty}\left|\phi_{j}-w_{j}^{0}\right|-\left|u_{+}-u_{-}\right| \\
& \leq \sum_{j=-\infty}^{\infty}\left|\phi_{j \pm 1}-w_{j}^{0}\right| \\
& \leq \sum_{j=-\infty}^{\infty}\left|\phi_{j}-w_{j}^{0}\right|+\left|u_{+}-u_{-}\right|
\end{aligned}
$$

and

$$
\begin{aligned}
& \sum_{j=-\infty}^{\infty}\left|\phi_{j+i}-w_{j}^{0}\right| \\
= & \sum_{j=-\infty}^{\infty}\left|\phi_{j}-w_{j}^{0}\right|-\left|\phi_{0}-w_{0}\right|+\left|\phi_{i}-w_{0}\right| \\
& -\sum_{j=-\infty}^{\infty} \operatorname{sign}(j)\left(\phi_{j}-\phi_{j+i}\right) \rightarrow \pm \infty
\end{aligned}
$$

as $i \rightarrow \pm \infty$. From these observations, we know that $C_{0} \leq\left|u_{+}-u_{-}\right|$.

By using the $l^{1}$-contracting property of Godunov's scheme, we estimate the $L^{1}$ norm of the approximation error as

$$
\begin{aligned}
& \sum_{j=-\infty}^{\infty}\left|u_{j}^{n}-w_{j}^{n}\right| \\
\leq & \sum_{j=-\infty}^{\infty}\left|u_{j}^{n}-\phi_{j}^{n}\right|+\sum_{j=-\infty}^{\infty}\left|\phi_{j}^{n}-w_{j}^{n}\right| \\
\leq & \sum_{j=-\infty}^{\infty}\left|w_{j}^{0}-\phi_{j}\right| \sum_{j=-\infty}^{\infty}\left|\phi_{j}^{n}-V_{j}^{n}\right|+\sum_{j=-\infty}^{\infty}\left|V_{j}^{n}-w_{j}^{n}\right| \\
\leq & \left(3+\frac{\lambda s(1-\lambda s)}{2 \gamma}\right)\left|u_{+}-u_{-}\right|+\sum_{j=-\infty}^{\infty}\left|V_{j}^{n}-w_{j}^{n}\right|
\end{aligned}
$$

where in the last step, we used Lemma 5.2. We observe that for all $j, V_{j}^{n}-w_{j}^{n}$ are of the same sign if not 0 . This yields that

$$
\begin{aligned}
& \sum_{j=-\infty}^{\infty}\left|V_{j}^{n}-w_{j}^{n}\right|=\left|\sum_{j=-\infty}^{\infty}\left(V_{j}^{n}-w_{j}^{n}\right)\right| \\
& =\left|\sum_{j=-\infty}^{\infty}\left(V_{j}^{n}-\phi_{j}^{n}\right)+\sum_{j=-\infty}^{\infty}\left(\phi_{j}^{n}-w_{j}^{n}\right)\right| \\
& \leq\left(3+\frac{\lambda s(1-\lambda s)}{2 \gamma}\right)\left|u_{+}-u_{-}\right|
\end{aligned}
$$


where we used the fact that

$$
\sum_{j=-\infty}^{\infty}\left(\phi_{j}^{n}-w_{j}^{n}\right)=\sum_{j=-\infty}^{\infty}\left(\phi_{j}-w_{j}^{0}\right) .
$$

Plunging (5.9) into (5.8), we obtain the result (5.3).

\section{ACKNOWLEDGEMENTS}

I would like to thank Professor Chi-Wang Shu at Brown University and Professor Hans Engler at Georgetown University for many discussions. My thanks also go to Dr. H.-S. Yu at the University of California at Los Angeles for many inspiring conversations.

\section{REFERENCES}

[EO] B. Engquist and S. Osher, One-sided difference approximations for nonlinear conservation laws, Math. Comp., 36 (1981), 321-351. MR 82c:65056

[EY] B. Engquist and Shih-Hsien Yu, Convergence of Lax-Wendroff scheme for piecewise smooth solutions with shocks, IMA preprint, (1994)

[Je] G. Jennings, Discrete shocks, Comm. Pure Appl. Math., 27 (1974) 25-37. MR 49:3358

$[\mathrm{Ku}] \mathrm{N}$. N. Kutnetsov, On stable methods for solving nonlinear first order partial differential equations in the class of discontinuous functions, Topics in Numerical Analysis III (Proc. Roy. Irish Acad. Conf.), J. J. H. Miller ed., Academic Press London, 1977, 183-197. MR 58:31874

[LX1] Jian-Guo Liu and Zhouping Xin, Nonlinear stability of discrete shocks for systems of conservation laws, Arch. Rat. Mech. Anal., 125 (1993) 217-256. MR 95c:35166

[LX2] Jian-Guo Liu and Zhouping Xin, $L^{1}$-stability of stationary discrete shocks, Math. Comp., 60 (1993) 233-244. MR 93d:35097

[Mi] D. Michelson, Discrete shocks for difference approximations to systems of conservation laws. Adv. Appl. Math., 5 (1984), 433-469. MR 86f:65159

[MR] A. Majda and J. Ralston, Discrete shock profiles for systems of conservation laws, Comm. Pure Appl. Math., 32 (1979) 445-482. MR 81i:35108

[OR] S. Osher and J. Ralston, $L^{1}$ stability of traveling waves with applications to convective porous media flow, Comm. Pure Appl. Math., 35 (1982) 737-749. MR 84j:35090

[Sm] Y. Smyrlis, Existence and stability of stationary profiles of the LW scheme, Comm. Pure Appl. Math., 43 (1990) 509-545. MR 91d:65143

[SX] A. Szepessy and Zhouping Xin, Nonlinear stability of viscous shock waves, Arch. Rat. Mech. Anal., 122 (1993) 53-103. MR 93m:35125

[Sz] A. Szepessy, On the stability of finite element methods for shock waves, Comm. Pure Appl. Math., 45 (1992) 923-946. MR 93f:65076

[TT] Tao Tang and Zhen-Huan Teng, The sharpness of Kuznetsov's $O(\sqrt{\delta x}) L^{1}$-error estimate for monotone difference schemes, Math. Comp. Vol 64 (1995), 581-589. MR 95f:65176

[TZ] Zhen-Huan Teng and Pingwen Zhang, to appear in SIAM J. Num. Anal.

[Yu] Shih-Hsien Yu, Stability of the traveling discrete shock profiles for Lax-Wendroff scheme, IMA preprint (1995).

Department of Mathematics, Georgetown University, Washington, DC 20057

E-mail address: fan@gumath1.math.georgetown.edu 